\title{
An Output Feedback Controller Design for Lateral Vehicle Dynamic
}

\author{
J. Bosche* A. El Hajjaji* \\ * MIS (Modélisation, Information et Systèmes), Université de Picardie \\ Jules Verne,7 rue du moulin neuf, 80000 Amiens, FRANCE (e-mail: \\ jerome.bosche@u-picardie.fr)
}

\begin{abstract}
This paper deals with the robust lateral control to improve vehicle dynamic behavior. Bounded variations on longitudinal speed, front and rear tire stiffnesses are considered allowing the vehicle model to form a polytope of linear systems. In fact, the vehicle model depends affinely on the longitudinal speed and its inverse. Taking this dependence into account, the polytope is associated with a hyper-trapezoidal domain. This representation of the polytope is more judicious and finally less conservative than a classical hyper-rectangular one. An algorithm is proposed to compute a static output feedback controller with reduced gains. The controller guaranteed a certain level of performance for the vehicle system in terms of stability, settling time and also robustness under a given class of uncertainty. In order to highlight the performance of the proposed control algorithm, a numerical simulation is performed.
\end{abstract}

Keywords: Vehicle Dynamics; Robust Control; LPV Systems; $\mathcal{L M I}$; Root-clustering.

\section{INTRODUCTION}

In automotive industry, considerable efforts continue to be made to improve the safety and comfort of passengers in dangerous driving situations by developing modern control systems. The ultimate goal is still to produce vehicles that anyone can drive "safely", "pleasantly" and "as one wishes". To achieve this, many control schemes are proposed in the literature. Recently, one popular method that has attracted more attention is robust control as it can guaranty both the robust stability and robust performance at the same time [1], [2], [3]. In this paper, a robust static feedback control design for vehicle lateral dynamics is proposed by considering lateral velocity as unavailable whereas longitudinal velocity and corner stiffness are variables. Moreover, to keep the output-feedback gain small, an algorithm is proposed. In the design, the Linear Parameter-Varying (LPV) polytopic model is adopted in order to take into account parametric variations. This polytopic model, inspired by [6], is quite original because it takes the affine dependency of the longitudinal speed and its inverse into account. Then a control strategy based on static output feedback is proposed to guarantee robust performances in terms of stability and comfort under critical driving conditions.

The precise objective of this work is to compute a matrix $K$, associated with a robust control law by static output feedback. The computed feedback gain has to stabilize the system while conferring to it a certain level of transient performances (settling time and damping ratio). The controller design procedure proposed in this work is based on a recent output feedback stabilization technique $[4,5]$. Conditions for robust stabilization are expressed in terms of Linear Matrix Inequality $(\mathcal{L} \mathcal{M I})$ involving ParameterDependent Lyapunov Matrix (PDLM).

The paper is organized as follows: in section 2 , the consid- ered model of vehicle is expressed in the state-space with polytopic uncertainties. Then the precise purpose is defined. A robust output-feedback $\mathcal{D}$-stabilization condition for such a Linear Time-Varying (LTV) system is proposed in section 3. Section 4 is dedicated to the algorithm used to compute a robust controller with reduced gains. It is then applied to the model of the vehicle presented in section 2 . Some conclusions are finally given in the last section.

Notations : We denote by $M^{\prime}$, the transpose conjugate of $M$, by $\mathcal{H}(M)$ the Hermitian expression $M+M^{\prime}$. In the hermitian matrices, notation $(\bullet)$ ' enables to avoid repeating the symmetric blocks. The Kronecker product is denoted by $\otimes$. $\|M\|_{2}$ is the 2 -norm of matrix $M$ induced by the Euclidean vector-norm, i.e. the maximal singular value of $M . \mathbb{I}_{n}$ is the identity matrix of order $n, \mathbb{O}$ is a null matrix of suitable dimension. Matrix inequalities are

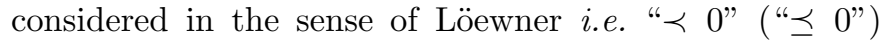
means negative (semi-)definite and " $\succ 0 "$ " " $\succeq 0 ")$ positive (semi-)definite. Small letters are used for scalar numbers and vectors while capital letters denote matrices or sets. At last, $\tilde{z}$ is the conjugate of the complex number $z=x+\mathbf{i} y$.

\section{PROBLEM STATEMENT}

\subsection{LTV vehicle model}

In [3], a simplified yaw-plane model of vehicle dynamics is used to describe the lateral dynamics of a highway vehicle in the state-space. The model considered in this work is quite similar. It corresponds to the following LTV equations:

$$
\left\{\begin{array}{l}
\dot{\mathbf{x}}(t)=A(t) \mathbf{x}(t)+B(t) \mathbf{u}(t) \\
\mathbf{y}(t)=C \mathbf{x}(t)
\end{array}\right.
$$


where $\mathbf{x}(t)=\left[\begin{array}{llll}v_{y} & r & y & \psi\end{array}\right]^{\prime} \in \mathbb{R}^{n}$ is the state vector. $v_{y}$ $r, y$ and $\psi$ are respectively the lateral velocity, the yaw rate, the lateral offset of the vehicle's center of gravity (CG) and the heading error of the vehicle as illustrated in Fig. 1.

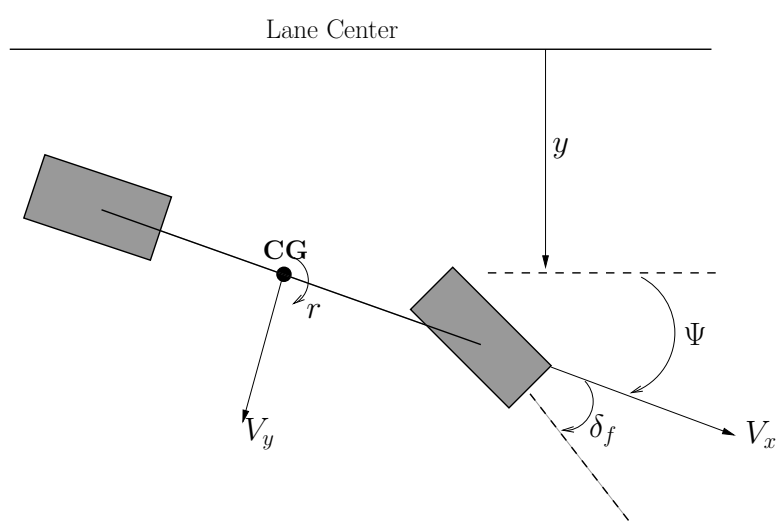

Figure 1. Vehicle variables definition

The front steering angle $\delta_{f}(t)=\mathbf{u}(t) \in \mathbb{R}^{m}$ is the command input.

$\mathbf{y}(t)=\left[\begin{array}{lll}r & y & \psi\end{array}\right]^{\prime} \in \mathbb{R}^{p}$ is the output vector. Indeed, the lateral speed $v_{y}$ is supposed not available for measure whereas other state measurements can be done with not very expensive sensors, such as inertial navigation equipment. Consequently, it comes

$$
C=\left[\mathbb{O}_{3,1} \mid \Pi_{3}\right] \text {. }
$$

$A(t) \in \mathbb{R}^{n \times n}$ and $B(t) \in \mathbb{R}^{n \times m}$ are respectively the timevarying state and input matrices given by (2) and (3) with the nominal parameter values of Tab. 1 .

$$
\begin{gathered}
A(t)=A\left(C_{f}(t), C_{r}(t), V_{x}(t), \Lambda_{x}(t)\right) \\
=\left[\begin{array}{cccc}
a_{11} & a_{12} & 0 & 0 \\
a_{21} & a_{22} & 0 & 0 \\
1 & 0 & 0 & a_{34} \\
0 & 1 & 0 & 0
\end{array}\right] \\
B(t)=B\left(C_{f}(t), C_{r}(t)\right)=\left[\begin{array}{llll}
b_{11} & b_{21} & 0 & 0
\end{array}\right]^{\prime}
\end{gathered}
$$

with

$$
\left\{\begin{array}{l}
a_{12}=-V_{x}(t)-\frac{2}{M} \Lambda_{x}(t)\left(a C_{f}(t)-b C_{r}(t)\right) \\
b_{11}=\frac{2 C_{f}(t)}{M}, \quad a_{11}=-\frac{2}{M} \Lambda_{x}(t)\left(C_{f}(t)+C_{r}(t)\right) \\
b_{21}=\frac{2 a C_{f}(t)}{I}, \quad a_{21}=-\frac{2}{I} \Lambda_{x}(t)\left(a C_{f}(t)-b C_{r}(t)\right) \\
a_{34}=V_{x}(t), \quad a_{22}=-\frac{2}{I} \Lambda_{x}(t)\left(a^{2} C_{f}(t)+b^{2} C_{r}(t)\right)
\end{array}\right.
$$

$V_{x}(t)$ is the longitudinal speed and $\Lambda_{x}(t)=\frac{1}{V_{x}(t)}$. In fact, it is supposed that

$$
V_{x}(t) \in\left[\underline{V_{x}} ; \overline{V_{x}}\right]
$$

Table 1. Vehicle Model Data

\begin{tabular}{|c|c|c|}
\hline Parameter & Value & Description \\
\hline a & $0.9637 \mathrm{~m}$ & distance from CG to front axle \\
\hline $\mathrm{b}$ & $1.7287 \mathrm{~m}$ & distance from CG to rear axle \\
\hline $\mathrm{M}$ & $1419 \mathrm{~kg}$ & vehicle mass \\
\hline$I$ & $2618 \mathrm{~kg} \cdot \mathrm{m}^{2} \mathrm{~m}$ & vehicle yaw moment of inertia \\
\hline
\end{tabular}

where $V_{x}$ and $\overline{V_{x}}$ are some constant values corresponding to the minimal and maximum speed which the vehicle can attain. Consequently, it comes

$$
\Lambda_{x}(t) \in\left[\underline{\Lambda_{x}} ; \overline{\Lambda_{x}}\right]=\left[\frac{1}{\overline{V_{x}}} ; \underline{\frac{1}{V_{x}}}\right]
$$

Also, to take road conditions into account, the front and rear cornering stiffness coefficients $\left(C_{f}(t)\right.$ and $\left.C_{r}(t)\right)$ such as:

$$
C_{f}(t) \in\left[\underline{C_{f}} ; \overline{C_{f}}\right] \text { and } C_{r}(t) \in\left[\underline{C_{r}} ; \overline{C_{r}}\right]
$$

where $\overline{C_{f}}$ and $\overline{C_{r}}$ correspond to some dry road conditions whereas $C_{f}$ and $\underline{C_{r}}$ can correspond to some more critical road conditions (wet or iced road).

According to (4), (5) and (6), the LTV model (1) is expressed on a following polytopic form such as $\mathbb{M}=$ $[\mathbb{A} \mid \mathbb{B}]$ belongs to a polytope of matrices $\mathcal{M}$ defined by:

$$
\begin{gathered}
\mathcal{M}=\left\{\mathbb{M}=M(\theta)=[A(\theta) \mid B(\theta)] \in \mathbb{R}^{n \times n+m} \mid\right. \\
\left.M(\theta)=\sum_{i=1}^{N}\left(\theta_{i}\left[A_{i} \mid B_{i}\right]\right) \cdot\right\}
\end{gathered}
$$

where $\theta \in \Theta$, the set of all barycentric coordinates:

$$
\Theta=\left\{\theta=\left[\begin{array}{c}
\theta_{1} \\
\vdots \\
\theta_{N}
\end{array}\right] \in\left\{\mathbb{R}^{+}\right\}^{N} \mid \sum_{i=1}^{N} \theta_{i}=1\right\} .
$$

Extreme matrices $M_{i}, i=1, \ldots, N$ are the vertices of $\mathcal{M}$. In a classical way, it can correspond to all possible combinations of the minimal and maximum values of $V_{x}(t), \Lambda_{x}(t), C_{f}(t)$ and $C_{r}(t)$, such as:

$$
\left\{\begin{array}{rl}
M_{1} & =\left[A\left(\underline{V_{x}}, \underline{\Lambda_{x}}, \underline{C_{f}}, \underline{C_{r}}\right) \mid B\left(\underline{C_{f}}, \underline{C_{r}}\right)\right] \\
M_{2} & =\left[A\left(\underline{V_{x}}, \overline{\Lambda_{x}}, \underline{C_{f}}, \underline{C_{r}}\right) \mid B\left(\underline{C_{f}}, \underline{C_{r}}\right)\right] \\
M_{3}= & {\left[A\left(\overline{V_{x}}, \underline{\Lambda_{x}}, \underline{C_{f}}, \underline{C_{r}}\right) \mid B\left(\underline{C_{f}}, \underline{C_{r}}\right)\right]} \\
M_{4}= & {\left[A\left(\overline{V_{x}}, \overline{\Lambda_{x}}, \underline{C_{f}}, \underline{C_{r}}\right) \mid B\left(\underline{C_{f}}, \underline{C_{r}}\right)\right]} \\
M_{5}= & {\left[A\left(\underline{V_{x}}, \underline{\Lambda_{x}}, \underline{C_{f}}, \overline{C_{r}}\right) \mid B\left(\underline{C_{f}}, \overline{C_{r}}\right)\right]} \\
\vdots \\
M_{16}=\left[A\left(\overline{V_{x}}, \overline{\Lambda_{x}}, \overline{C_{f}}, \overline{C_{r}}\right) \mid B\left(\overline{C_{f}}, \overline{C_{r}}\right)\right]
\end{array} .\right.
$$

The polytope is then described as a hyper-rectangle with four dimensions. However, for such a system, this extrapolation is not judicious and quite conservative. Indeed, let us focus our attention on the hyperbole of Fig. 2. It represents 
the variation of $\Lambda_{x}(t)$ according to $V_{x}(t)$ in a bounded domain of variation. It is clear that the choice of the extreme matrices $M_{i}$ given in (9) is not judicious. Indeed, the corresponding extrapolation is the rectangular domain MNOP. However, it is better to choose the polytope as small as possible. For this reason, the trapezoidal domain $M O R Q$ shown on Fig. 2. is preferred [6].

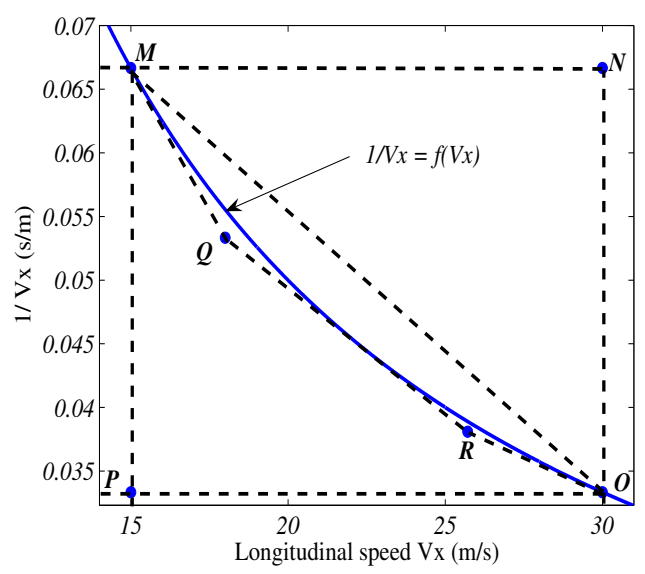

Figure 2. Structure of the polytope

The new vertices are

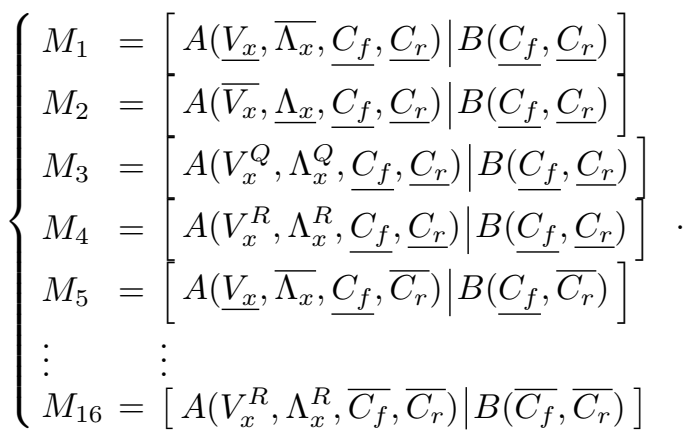

with

$$
\left\{\begin{aligned}
V_{x}^{Q} & =2 \underline{\underline{V_{x}}} \frac{\sqrt{\overline{V_{x}}}}{\sqrt{\frac{V_{x}}{2}}+\sqrt{\overline{V_{x}}}} \\
\Lambda_{x}^{Q} & =\frac{\underline{V_{x}}+\sqrt{\frac{V_{x} \overline{V_{x}}}{\sqrt{V_{x}}}}}{\sqrt{\frac{V_{x}}{2}+\sqrt{\overline{V_{x}}}}} \\
V_{x}^{R} & =2 \overline{V_{x}} \frac{\sqrt{V_{x}}}{\overline{V_{x}}+\sqrt{\underline{V_{x}}}}
\end{aligned}\right.
$$

\subsection{Precice purpose}

The main objective of this work is to compute a matrix $K$, associated with a robust control law by static output feedback, so as to guarantee the system (1) some transient performances.

Transient performances Some transient performances are strongly influenced by the location of the state matrix spectrum of the closed-loop system in the complex plane, in terms of settling time, damping ratio... One of the main goal of this work is to place the all spectrum in a region of the complex plane, noted $\mathcal{D}$. Here, the region $\mathcal{D}$ corresponds to a ellipsoidal matrix inequality-region [7] of degree 1 , described by

$$
\mathcal{D}=\left\{z \in \mathbb{C} \mid r_{00}+r_{10} z+r_{10}^{\prime} \tilde{z}+r_{11} z \tilde{z} \prec 0\right\}
$$

where

$$
R=\left[\begin{array}{ll}
r_{00} & r_{10} \\
r_{10}^{\prime} & r_{11}
\end{array}\right] \in \mathbb{R}^{2 \times 2}
$$

is a symmetric matrix and $r_{11} \geq 0 \in \mathbb{R}$. $\mathcal{D}$ corresponds to a a shifted vertical half plane or a disc centered on the real axis of the complex plane. When the whole spectrum of the state matrix is strictly clustered in a specified region $\mathcal{D}$, the system (or the state matrix) is said $\mathcal{D}$-stable.

Control objective The control system configuration is shown in Fig. 3. The closed-loop model is then described by:

$$
\left\{\begin{array}{l}
\dot{\mathbf{x}_{c l}}(t)=A(\theta) \mathbf{x}_{c l}(t)+B(\theta) K \mathbf{y}(t)+B(\theta) \delta_{f}(t) \\
\mathbf{y}(t)=C \mathbf{x}_{c l}(t)
\end{array}\right.
$$

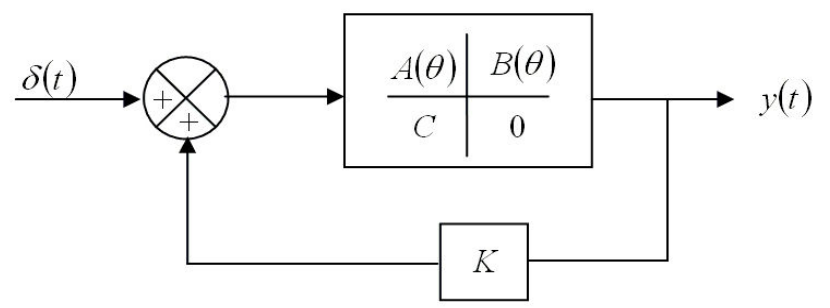

Figure 3. Closed-loop model

Let us consider

$$
\mathbb{A}_{c l}=A_{c l}(\theta)=A(\theta)+B(\theta) K C,
$$

the closed-loop state matrix of model (13) and $\lambda\left(\mathbb{A}_{c l}\right)$ the set of the eigenvalues $\mathbb{A}_{c l}$. The precise control objective is to compute $K$ such as $\lambda\left(\mathbb{A}_{c l}\right) \in \mathcal{D}$.

\section{MAIN RESULT}

This part proposes a method to compute $K$, based on an oiginal idea of [4], associated with a robust control law by output feedback for the multivariable linear system defined in (13), allowing to achieve the objectives presented in the preceding section.

This idea consists in finding a state feedback and an output feedback checking the same robust $\mathcal{D}$-stability property through the existence of the same set of Lyapunov matrices. It amounts to solving the following $\mathcal{L} \mathcal{M} \mathcal{I}$ system

$$
\left\{\begin{array}{l}
\mathcal{R}\left(\mathbb{A}_{s}, \mathbb{P}\right) \prec 0 \\
\mathcal{R}\left(\mathbb{A}_{c l}, \mathbb{P}\right) \prec 0
\end{array}\right.
$$


where $\mathcal{R}\left(\mathbb{A}_{\alpha}, \mathbb{P}\right)$ ( $\alpha$ denoting either $s$ or $c l$ ) is a sufficient $\mathcal{D}$-stability condition for $\mathbb{A}_{\alpha}$ with

$$
\mathcal{M}_{\alpha}\left(\mathbb{A}_{\alpha}, \mathbb{P}\right)=\left(r_{00} \mathbb{P}+\mathcal{H}\left(r_{10} \mathbb{P} \mathbb{A}_{\alpha}\right)+r_{11} \mathbb{A}_{\alpha}^{\prime} \mathbb{P} \mathbb{A}_{\alpha}\right)
$$

$\mathbb{A}_{s}$ and $\mathbb{A}_{c l}$ are respectively the state and the output closed-loop state matrices. $\mathbb{A}_{c l}$ is defined in (14) whereas $\mathbb{A}_{s}$ is given by

$$
\mathbb{A}_{s}=\mathbb{A}+\mathbb{B} K_{s} .
$$

where $K_{s}$ is associated with state feedback.

A sufficient condition checking $\mathcal{L} \mathcal{M I}$ system (15) is now proposed.

Theorem 1. Let a system desbribed by (13) with (14). Let also consider a state feedback matrix $K_{s}$ which $\mathcal{D}$ stabilizes the pair $(\mathbb{A}, \mathbb{B})$, i.e. $\lambda\left(\mathbb{A}_{s}=\mathbb{A}+\mathbb{B} K_{s}\right) \in \mathcal{D}$. Then,

$$
K=G^{-1} H
$$

is an output feedback matrix with reduced gains which $\mathcal{D}$-stabilizes the system (13) if the $\mathcal{L} \mathcal{M I}$ optimization problem (19) is satisfied.

$$
\min _{\left\{\epsilon, P_{1}, . ., P_{N}, F, G, H, Q>0\right\}} \epsilon
$$

subject to

$$
\begin{aligned}
Z_{i} & \prec 0 \quad \forall i \in\{1, . ., N\} \\
\text { and } S(\epsilon, H) & =\left[\begin{array}{cc}
\epsilon \mathbb{I}_{p} & H^{\prime} \\
H & Q
\end{array}\right] \succ 0
\end{aligned}
$$

where $\gamma \in \mathbb{R}, P_{i}=P_{i}^{\prime} \in \mathbb{R}^{n \times n} \forall i \in\{1, . ., N\}, G \in \mathbb{R}^{m \times m}$ is a nonsingular matrix, $F \in \mathbb{R}^{(2 n+m) \times n}, H \in \mathbb{R}^{m \times p}$, $Q>0 \in \mathbb{R}^{m \times m}$ and

$$
\begin{aligned}
Z_{i}= & {\left[\begin{array}{c|c}
R \otimes P_{i} & \mathbb{O} \\
\hline \mathbb{O} & \mathbb{O}
\end{array}\right]+\mathcal{H}\left\{F\left[A_{s_{i}}\left|-\mathbb{I}_{n}\right| B_{i}\right]\right\} } \\
+ & \mathcal{H}\left\{\left[\frac{\mathbb{O}_{2 n, m}}{\mathbb{I}_{m}}\right] G\left[-K_{s}|\mathbb{O}|-\mathbb{I}_{m}\right]\right\} \\
& +\mathcal{H}\left\{\left[\frac{\mathbb{O}_{2 n, m}}{\mathbb{I}_{m}}\right] H[C \mid \mathbb{O}]\right\} \prec 0 .
\end{aligned}
$$

Remark 1. Minimizing $\epsilon$ in (20b) results in minimizing the norm of $H$, and consequently the one of $K$.

\section{Proof :}

For any vector of barycentric coordinates $\theta \in \Theta$, let us consider the convex combination

$$
\mathbb{Z}=\sum_{i=1}^{N} \theta_{i} Z_{i}
$$

Since all $\theta_{i}$ are positive, it comes:

$$
\mathbb{Z} \prec 0 \text {. }
$$

Let us define the matrix $S=K C-K_{s}$, the condition (22) becomes with (18):

$\mathbb{Z}=\left[\begin{array}{c|c}R \otimes \mathbb{P} & \frac{S^{\prime} G^{\prime}}{\mathbb{O}} \\ \hline G S \mid \mathbb{O} & -\left(G+G^{\prime}\right)\end{array}\right]+\mathcal{H}\left\{F\left[\mathbb{A}_{s}\left|-\mathbb{I}_{n}\right| \mathbb{B}\right]\right\} \prec 0$.

with

$$
\left\{\begin{array}{l}
\mathbb{P}=\sum_{i=1}^{N} \theta_{i} P_{i} \\
\mathbb{A}_{s}=\sum_{i=1}^{N} \theta_{i} A_{s_{i}}=\sum_{i=1}^{N} \theta_{i}\left(A_{i}+B_{i} K_{s}\right)
\end{array}\right.
$$

Now define matrix $\mathcal{N}_{f}$ by

$$
\mathcal{N}_{f}=\left[\begin{array}{c|c|c}
\mathbb{I}_{n} & \mathbb{A}_{s}^{\prime} & \mathbb{O} \\
\hline \mathbb{O} & \mathbb{B}^{\prime} & \mathbb{I}_{m}
\end{array}\right]
$$

the left orthogonal complement of $\left[\mathbb{A}_{s}\left|-\mathbb{I}_{n}\right| \mathbb{B}\right]^{\prime}$.

Then according to the elimination lemma [8], (23) can be written as follows:

$$
\mathcal{N}_{f}^{\prime}\left[\begin{array}{c|c}
R \otimes \mathbb{P} & \frac{S^{\prime} G^{\prime}}{\mathbb{O}} \\
\hline G S \mid \mathbb{O} & -\left(G+G^{\prime}\right)
\end{array}\right] \mathcal{N}_{f} \prec 0
$$

After simple algebraic manipulations, (24) is equivalent to

$$
\mathbb{Z}=\left[\begin{array}{c|c}
\mathcal{R}\left(\mathbb{A}_{s}, \mathbb{P}\right) & r_{10} \mathbb{P B}+r_{11} \mathbb{A}_{s}^{\prime} \mathbb{P B}+S^{\prime} G^{\prime} \\
\hline(\bullet)^{\prime} & r_{11} \mathbb{B}^{\prime} \mathbb{P B}-G-G^{\prime}
\end{array}\right] \prec 0 .
$$

Let consider $\mathcal{N}_{k}$ and $\mathcal{N}_{g}$ defined by

$$
\left\{\begin{array} { l } 
{ \mathcal { N } _ { k } = [ \Pi _ { n } | \mathbb { O } | \mathbb { O } ] } \\
{ \mathcal { N } _ { g } ^ { \prime } = [ \begin{array} { c | c | c } 
{ \mathbb { I } _ { n } } & { S ^ { \prime } } & { \mathbb { O } } \\
{ \hline \mathbb { O } } & { \mathbb { O } } & { \mathbb { I } _ { m } }
\end{array} ] }
\end{array} \text { such as } \left\{\begin{array}{l}
\mathcal{N}_{k}\left[\mathbb{O}\left|\Pi_{n}\right| \mathbb{O}\right]^{\prime}=\mathbb{O} \\
\mathcal{N}_{g}^{\prime}\left[S\left|-\Pi_{n}\right| \mathbb{O}\right]^{\prime}=\mathbb{O}
\end{array}\right.\right.
$$

Based on the elimination lemma, (25) is equivalent to:

$$
\begin{aligned}
& \mathcal{N}_{k} \Psi \mathcal{N}_{k}^{\prime} \prec 0 \\
& \mathcal{N}_{g}^{\prime} \Psi \mathcal{N}_{g} \prec 0
\end{aligned}
$$

(26a) is nothing but $\mathcal{R}\left(\mathbb{A}_{s}, \mathbb{P}\right)$ once developed where (26b) leads to $\mathcal{R}\left(\mathbb{A}_{c l}, \mathbb{P}\right)$.

\section{ALGORITHM}

This section proposes an algorithm in order to compute a robust control law by static output feedback with reduced gains. It aims at $\mathcal{D}$-stabilizing the triplet $(A(\theta), B(\theta), C)$ of system (13). The resolution method that we propose is as follows: 
Step 1 : First at all, it is advisable to define matrix $R$ corresponding to the $\mathcal{D}$-region described by (11) according to the desired transient performances, in terms of settling time or damping ratio.

Step 2 : It is then question to compute several statefeedback $K_{s}^{j}(j \in\{1, \ldots, \bar{j}\})$, each $\mathcal{D}$-stabilizing the pair $(\mathbb{A}, \mathbb{B})$. With this intention, a specified eigenstructure assignment technique will be used in this work [9]. It exploits the degree of freedom on the eigenvalues and eigenvectors. A genetic algorithm begins with a set of parameters, associated with $\bar{j}$ static state feedback $K_{s}^{j}$. It constitutes the initial population.

Step 3 : Solve the $\mathcal{L} \mathcal{M I}$ optimization problem (19) $\forall j \in\{1, . ., \bar{j}\}$, subject to (20a) and (20b).

Step 4 : The best set of parameters corresponding to the smallest values of $\kappa^{j}=\left\|K^{j}\right\|_{2}$, is selected and used to form many others parameters at least better than the old ones, with recombination and mutation techniques.

Test : Then we can define a convergence criterion which, if it is satisfied, a priori picks up the "best", $K$ (denoted by $K^{\diamond}$ ) in the sense of the criterion $\kappa$, and stops the process. If the convergence test does not hold, an evolution process based on genetic algorithms is used to improve the control law, iteration after iteration, until the convergence criterion is finally satisfied and leads to $K^{\diamond}$.

\section{NUMERICAL ILLUSTRATION}

The numerical illustration considers the unstable vehicle model defined in section 2.1 with the values given in Tab. 1. The computations are performed with MATLAB 7.1 and its LMIToolbox [10] on a PC Pentium 2930 Mhz.

As in [3], the clustering region $\mathcal{D}$ considered in this example is a vertical half-plane defined by $x<-0.65$.

In a first time, a nominal case is considered with

$$
\left\{\begin{array}{l}
V_{x}(t)=V_{x_{0}}=20 \mathrm{~m} . \mathrm{s}^{-1}, \\
C_{f}(t)=C_{f_{0}}=56600 \text { N.rad } \\
C_{r}(t)=C_{r_{0}}=63500 \text { N.rad }
\end{array}\right.
$$

A static output feedback is then computed according to this linear time-invariant model. It leads to:

$$
K_{L}=[-0.0635-0.1064-0.2307] \text {. }
$$

Then longitudinal speed variations and road conditions are taken into account such as

$$
\left\{\begin{array}{l}
V_{x}(t) \in\left[15 m . s^{-1} ; 40 m . s^{-1}\right], \\
C_{f}(t) \in\left[28000 N . r a d^{-1} ; 56600 N . r a d^{-1}\right] \\
C_{r}(t) \in[31500 N . r a d
\end{array}\right.
$$

The matrix $\mathbb{M}$ defined in (7) is then assumed to belong to a polytope of matrices with 16 vertices.
According to this, two other static output feedback matrices $\left(K_{R} \diamond\right.$ and $\left.K_{T} \diamond\right)$ are computed by solving the $\mathcal{L} \mathcal{M} \mathcal{I}$ optimization problem (19) and using the genetic algorithm recalled in section 4 . This algorithm considers an initial population of 50 individuals $(\bar{j}=50)$ and a convergence criterion defined such as $\kappa=\left\|K^{j}\right\|_{2} \leq 10$. The first output feedback matrix $\left(K_{R} \diamond\right)$ is calculated by considering the polytopic representation (9) of the LTV model (1), i.e. a hyper-rectangular domain. The second one $\left(K_{T} \diamond\right)$ is computed by considering the polytopic representation (10), i.e. a hyper-trapezoidal domain. It leads to

and

$$
K_{R}^{\diamond}=[-0.4444-0.2740-3.6275]
$$

$$
K_{T}^{\diamond}=[-0.8346-0.4535-6.8212] .
$$

Remark 2. To emphasize the "hyper-trapezoidal" polytopic representation (10), a larger variation domain of the longitudinal speed is considered such as:

$$
V_{x}(t) \in\left[15 m . s^{-1} ; 45 m . s^{-1}\right] .
$$

$C_{f}(t)$ and $C_{r}(t)$ are defined as in (27). According to this, the polytopic representation (10) allows the optimization problem (19) to be solved with the following output feedback matrix

$$
K_{T 2}^{\diamond}=[-0.5752-0.3718-5.2912]
$$

whereas it is not possible with the representation based on (9).

Furtheremore, in order to confirm the effectiveness of the control technique designed in this study, the following computer simulations are considered. It corresponds to a slalom test (ISO double lane change). $\delta_{f}$ changes from 5 to -5 degrees whereas the longitudinal speed $\left(V_{x}\right)$ varies between 15 and $40 \mathrm{~m} . \mathrm{s}^{-1}$. Bad road conditions are also considered between $t=6 \mathrm{~s}$ and $t=8 \mathrm{~s}$. It corresponds to a reduction about $50 \%$ of the front and rear cornering stiffness coefficients $\left(C_{f}\right.$ and $\left.C_{r}\right)$.

The resulting state signals $v_{y}, r, y$ and $\psi$ are plotted for different values of $K\left(K_{L}, K_{T}^{\diamond}\right.$ and $\left.K_{R}^{\diamond}\right)$. The front and rear forces $\left(F_{f}\right.$ and $\left.F_{r}\right)$, the slip angle $(\beta)$ and the lateral acceleration $\left(a_{y}\right)$ are also represented.

Fig. 4 shows the simulation results for $K=K_{T}^{\diamond}$ (solid line) and $K=K_{R}^{\diamond}$ (dotted line). It emphasizes the quality of these two output feedback control laws. We can just remark that $K_{T}^{\diamond}$ confers a better level of performance, especially for the lateral offset $y$. On Fig. 5, the same maneuver is considered but this time, the static output feedback associated with matrix $K_{T}^{\diamond}$ (solid line) is compared to the linear control associated with matrix $K_{L}$ (dotted line). It is clear that this linear control is not efficient, notably in case of bad road conditions.

\section{CONCLUSIONS}

In this paper, a robust output feedback stabilization procedure for the lateral control of a vehicle is proposed. The considered vehicle model is LPV and then described 

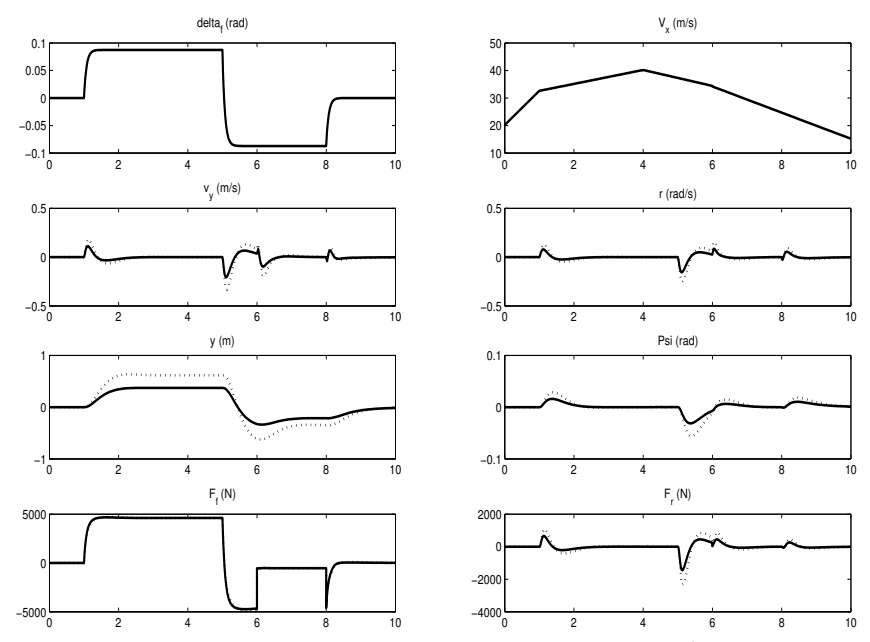

beta(rad)
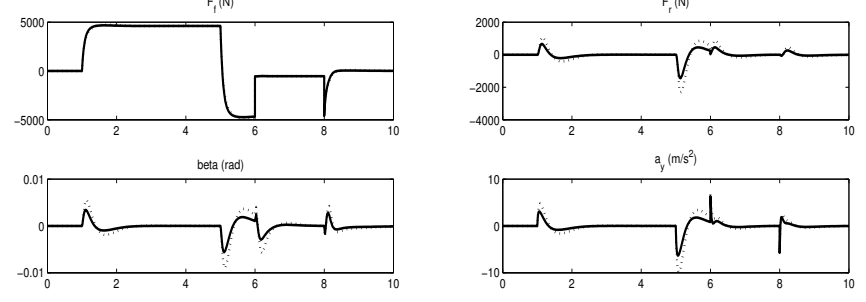

Figure 4. Vehicle response with $K=K_{T}^{\diamond}(-)$ and $K_{R}^{\diamond}(.$.
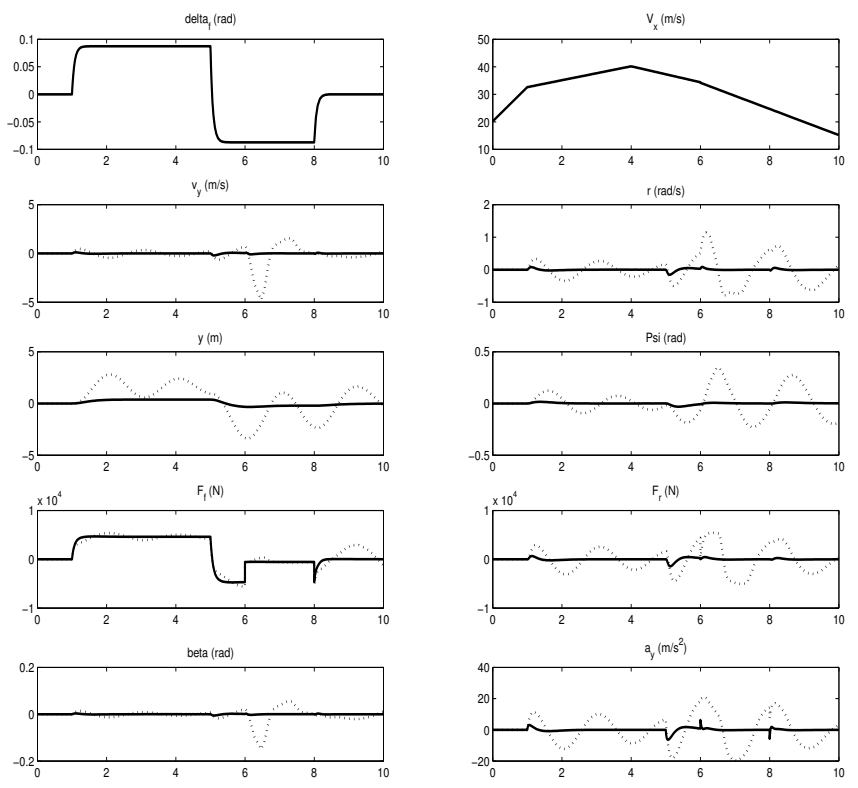

Figure 5. Vehicle response with $K=K_{T}^{\diamond}(-)$ and $K_{L}(.$.

as a convex combination of linear systems. An algorithm using recent results of control theory is proposed to design a robust static output-feedback controller with reduced gains. It improves the robust performances of the control law and provides some desired vehicle performances like a greater maneuverability for passenger vehicles.

\section{REFERENCES}

[1] J.Ackermann, Modeling and control of car yaw and lateral dynamics with uncertain non-lineartire characteristics, Proceedings 6th Biennial Conference on
Engineering Systems Design and Analysis, Istanbul, July 2002.

[2] R. E. Benton Jr and D. Smith. Output feedback stabilization with prescribed degree of stability. Proceedings of the American Control Conference, pp 499504, Albuquerque, New Mexico, June 1997.

[3] R. E. Benton Jr and D. Smith. A static-outputfeedback design prodedure for robust emergency lateral control of a highway vehicle. IEEE Transactions on Automatic Control, vol. 13(4), pp 618-623, July 2005.

[4] D. Mehdi, E. Boukas and O. Bachelier. Static output feedback design for uncertain linear discrete time systems. IMA Journal of Mathematical Control and Information, vol. 21(1), pp 1-13, 2004.

[5] J. Bosche and A. El Hajjaji. Robust static output feedback for vehicle dynamics. International Conference on Advances in Vehicle Control and Safety, Buenos Aires, Argentina, February 2007.

[6] L. Palladino, G. Duc and R. Pothin. LPV control for mu-split braking assistance of a road vehicle. 44th IEEE Conference on Decision and Control and European Control Conference, ECC 2005, Sville, Espagne, dcembre 2005.

[7] D. Peaucelle, D. Arzelier, O. Bachelier, and J. Bernussou. A new robust $\mathcal{D}$-stability condition for real convex polytopic uncertainty.Systems and Control Letters, 40(1):21-30, May 2000.

[8] R. E. Skelton, T. Iwasaki, et K. Grigoriadis, A unified approach to linear control design, Taylor and Francis series in Systems and Control, 1997.

[9] B. C. Moore, "On the flexibility offered by state feedback in multivariable systems beyond closed loop eigenvalue assignment," IEEE Transactions on Automatic Control, vol. 21, pp. 689-692, 1976.

[10] P. Gahinet and A. Nemirovski and A.J. Laub and M. Chilali,LMI Control Toolbox, The MathWorks, Inc., 1995. 